R Spiller, ${ }^{1}$ C Swan, ${ }^{2}$ E Campbell, ${ }^{1}$ M Hastings, ${ }^{3}$ G Dukes, ${ }^{4}$ P Whorwell, ${ }^{3}$ I Hall ${ }^{2}$, N Duroudier* ${ }^{1}$ NIHR BRU NDDC, University of Nottingham, Nottingham, UK; ${ }^{2}$ Department of Therapeutics and Molecular Medicine, University of Nottingham, Nottingham, UK; ${ }^{3}$ Neurogastroenterology, Wythenshawe Hospital, Manchester, UK; ${ }^{4}$ Academic DPU, GlaxoSmithKline, Harlow, UK

Introduction The heritability of irritable bowel syndrome (IBS) has been estimated at approximately $50-60 \%$. An important subgroup (18\%) of all IBS patients develop symptoms after gastrointestinal infection (postinfective IBS, PI-IBS). PI-IBS develops in 10\% of Campylobacter jejuni infection, 2/3 of which are of the IBS with diarrhoea subtype (IBS-D).

Aims To use changes in rectal mucosal gene expression following $C$ jejuni infection which were also seen in irritable bowel syndrome as a guide to identify and test candidate genes in IBS.

Methods Study 1: 31 patients with documented C. jejuni infection, 37 IBS with diarrhoea and 25 healthy volunteers provided blood and rectal biopsies for mRNA assessment using an Affymetrix array. Genes showing $>1.5$-fold change in both Group 1 and 2 compared with HV were validated by qPCR. Polymorphisms of these candidate gene were assessed in 168 IBS-D, 132 IBS with constipation (IBS-C) and 153 healthy volunteers (HV).

Results As previously reported ${ }^{1}$ the following proinflammatory genes were increased in rectal biopsies following $C$ jejuni infections and in IBS-D: CCL11, CCL13, TNFSF15, Calpain and GABRE while NR1D1 and GPR161 were depressed. We found no difference in the distribution of genetic polymorphisms of the CCL11, CCL13, genes between HV and IBS. We found an increased frequency of the $\mathrm{T}$ allele in the TNFSF15 SNP rs 6478108 in IBS-D ( $72 \%$ versus $62 \%$ in HV, $p=0.007$ ) but not IBS- $C$, in keeping with the $T$ allele being associated with an increased risk of Crohn's disease. Similarly we found increased frequency of the proinflammatory $\mathrm{C}$ allele from the TNFSF15 SNP rs7848647 and the G allele from TNFSF15 SNP rs6478109 in D-IBS ( $p=0.007$ and $p=0.015$ respectively). Although owing to small numbers we found a non-significant difference in TNFSF15 gene expression associated with the risk alleles $(p=0.1)$ there was a linear test for trend for the CC genotype of rs6478108 to have a lower TNFSF15 expression.

Conclusion We have identified a proinflammatory genetic tendency for increased TNFSF15 expression which may predispose to the development of IBS with diarrhoea.

Competing interests R. Spiller Grant/Research Support from: Norgene, Consultant for: Boehringer, Alberio, Conflict with: Study supported by grant from GSK, C. Swan: None Declared, E. Campbell: None Declared, M. Hastings: None Declared, G. Dukes Employee of: GSK, P. Whorwell: None Declared, I. Hall: None Declared.

Keywords genetics, Inflammation, Irritable Bowel Syndrome.

\title{
REFERENCE
}

1. Spiller et al. Gut 2009;58(Suppl 1):A30.

\section{PWE-085 IDENTIFYING AND TESTING CANDIDATE GENES UNDERLYING THE INFLAMMATORY BASIS OF IRRITABLE BOWEL SYNDROME}

